\title{
Topology optimization of the multi-fasteners jointed structure considering fatigue constraints
}

\author{
Jie Hou ${ }^{*}$, Jihong Zhu, Jie Wang, and Weihong Zhang \\ Engineering Simulation and Aerospace Computing, Northwestern Polytechnical University, 710072 Xi'an, Shaanxi, PR China
}

Received: 5 December 2017 / Accepted: 27 March 2018

\begin{abstract}
This paper is to present an important issue of fatigue failure in the design of multi-fasteners jointed structure. To avoid failure in the connection area, Sines criterion is utilized. Fatigue constraints are handled in the context of stress based topology optimization. To eliminate the high stresses caused by the finite element modeling, the control volume is defined to evaluate the stress states around the fasteners. The standard topology optimization is extended to minimize the structural compliance with fatigue failure constraints. To address singularity problems related to stress constraints, $q-p$ relaxation is used. P-norm is used as the constraints aggregation scheme. Basing on the above, the design sensitivity of fatigue constraints is derived and calculated. The proposed method is verified by a numerical example of an assembled I-beam. The comparisons of the numerical results have shown the effect of the fatigue constraint.
\end{abstract}

Keywords: Topology optimization / Multi-fastener Joint / Fatigue constraint

\section{Introduction}

The main idea of topology optimization is that the layouts and topology configurations of structures are considered as deign variables and optimized numerically to satisfy different design requirements. With its developments in engineering practice, topology optimization is consistently extended to tackle the increasing design complexity and the challenge of the practical application. Nowadays, it is proved to be a powerful tool for designers to generate the conceptual design beyond the limits of experience and knowledge.

Fatigue is a critical factor for the design of mechanical parts in fields of application. In an assembled aircraft structure, fasteners as bolts, rivets and the like, are widely used to joint the member components and distribute loads. Considering the structural strength, connections are the weakest point due to the high stress intensity, and most likely to fail. Past experience shows that $85 \%$ of fatigue failure in aircraft structures occurred around fasteners.

When strength is considered in topology optimization, stress and fatigue are usually the primary concerns. According to the cycle times, fatigue can be sorted into low-cycle fatigue (LCF) and high-cycle fatigue (HCF). When cycle times are less than $10^{4}$, it is LCF. The fatigue mechanism of LCF is governed by the plasticity and is enhanced by the stress level. Due to the complicated

\footnotetext{
* e-mail: houjie@mail.nwpu.edu.cn
}

fatigue mechanism, LCF has been focused in a few optimization studies. Desmorat and Desmorat [1] maximized the fatigue life considering elasto-plastic low-cycle fatigue and Lemaitre damage evolution law by topology optimization. Mrzyglod and Zielinski [2] used modified Neuber formula for approximate estimation of non-linear deformations in LCF and Ramberg-Osgood material for LCF damage to tackle the low-cycle fatigue constraints. For HCF, cycle times exceed $10^{4} \sim 10^{5}$ and the stress is relatively low. The fatigue mechanism is governed by stress states. For HCF, Mrzyglod and Zielinski [3] optimized mechanical structures working in high cycle load condition with evolutionary algorithms. Different fatigue criteria are implementation and evaluated including Sines, Crossland, and Dang Van criterion. In the work of Kaya et al. [4], a failed clutch fork was investigated and optimized by the response surface method to obtain a design with a lower von Mises stress. The fatigue analysis was made using the software MSC Fatigue. Sherif et al. [5] studied a minimum mass problem with a constraint on the upper bound of damage. The transient dynamic systems are solved equivalently by static load method. Holmberg [6] combined fatigue software with clustered principal stress constraints to generate an optimized design with minimum mass that can withstand prescribed loading conditions during the service life. The fatigue constraint was converted to the limits on stress by Palmgren-Miner's rule. Maxime et al. [7] used Sines equivalent mean and alternating stresses to address the fatigue resistance for an infinite life time design (Tab. 1). 
Earlier studies were focused on developing analytical and numerical methods for stress and failure predictions of multi-fastener joints. Typical models concerned panels joined by single or multiple joints, in which joint loads as well as stress distributions around pin holes, etc., are mostly analyzed.

For optimization, Zhang and Taylor [8] optimized the positions of two spot welds in a tensile shear specimen. They formulated two optimization problems: one was to maximize the stiffness of the structure under fatigue life constraints and the other was to seek the maximum fatigue life of the structure. The objective function to be minimized was the maximum radial stress, which was assumed to be correlated with the fatigue life. They used the so-called umbrella model of spot welds and the radial stress around a spot weld. Rui et al. [5] used an optimization algorithm to minimize the stress intensity factor of spot-welds, which was assumed to be a control parameter for the fatigue life of the structure. Ekh and Schön [9] evaluated the effects of different parameters on the load distribution, such as the mismatch of member plates, length of the overlap region and the fastener's stiffness. Optimization was then carried out to minimize the bearing stress. Bianchi et al. [10] developed an optimization procedure maximizing the loadcarrying capability of the joint system to balance the number and size of bolts for anti-fatigue design. In the work of Oinonen et al. [11], a "weakest link" method was proposed to optimize the layout of fasteners for the bracket-to-beam joints. The design objective was to ameliorate Von-Mises equivalent strain as well as shear loads in the joints. Poon and Xiong [12] considered the optimization of fastener joint locations, ply angles and stacking sequences of laminates, fastener diameters and edge distances, etc., to avoid the failure of fasteners.

In this paper, topology optimization is extended to the design of the assembled structure with fatigue constraints. The widely accepted high-cycle fatigue creation, Sines creation is implemented in the context of stress-based problems. The p-norm and $q p$-relaxation are used to circumvent the numerical issues. With all the proposed assumptions, sensitivities are derived by chain rule. Finally, the fatigue optimization of an assembled structure is presented.

\section{Fatigue in the assembled structure}

\subsection{Fatigue analysis}

Fatigue analysis is a critical issue in the design process of mechanical parts. Generally speaking, S-N curve is used to describe the fatigue life of the material under uniaxial high cycle fatigue loading. S-N curve based method like nominal stress method and stress severity factor method are commonly used in fatigue life analysis of aircraft structure. In practice, mechanical structures mostly serve under the multi-axial fatigue loading. In safe-life and damage tolerance design, a reliable fatigue strength assessment under multi-axial loading condition is necessary. Many researchers proposed different criteria to evaluate the fatigue behavior of the structure, like Sines, Crossland, Dang Van, etc. Those fatigue criteria can be sorted as

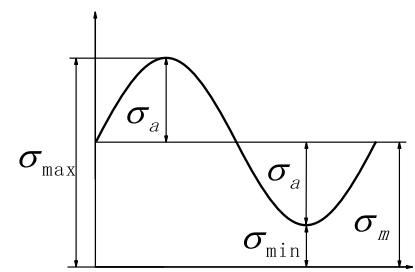

Fig. 1. Cyclic stress diagram of the sinusoidal load.

stress-based, strain-based, and energy based. In this paper, we adopt the Sines fatigue creation which is proposed in the 1950 s by Sines and considered as the first widely accepted fatigue criterion. The loading condition is considered as a typical harmonic load which is shown in Figure 1.

The magnitude of the stress state can be divided into

$$
\sigma_{\text {tot }}=c_{a} \sigma_{a}+c_{m} \sigma_{m}
$$

where $\sigma_{\text {tot }}$ is the global stress state, $c_{a} \sigma_{a}$ is the alternate component, and $c_{m} \sigma_{m}$ is the mean component. $0 \leq c_{a}$, $c_{m} \leq 1$ and $c_{a}+c_{m}=1$. With the stress state decomposition and linear elastic assumption, the alternate and mean stress components under the harmonic load can be derived simultaneously.

According to [13], Sines fatigue criterion can be written as:

$$
\begin{gathered}
\sqrt{J_{2, a}}+\kappa \cdot \sigma_{H, m} \leq \lambda, \\
\lambda=t_{-1}, \\
\kappa=\frac{6 t_{-1}}{f_{0}}-\sqrt{6} \text { or } \kappa=\frac{3 t_{-1}\left(\sigma_{f}+f_{-1}\right)}{\sigma_{f} f_{-1}}-\sqrt{6} .
\end{gathered}
$$

where $J_{2, a}$ is the second invariant of the deviatoric stress tensor, $\sigma_{H, m}$ is hydrostatic stress, $t_{-1}$ is the fatigue limit for reversed torsion, $f_{0}$ is the fatigue limit for repeated bending, $f_{-1}$ is the fatigue limit for reversed bending and $\sigma_{f}$ is the ultimate tensile strength. $\sigma_{H, m}$ can be determined as

$$
\sigma_{H, m}=\frac{1}{3} J_{1, m},
$$

where $J_{1, m}$ is the first stress invariant. Alternatively, equivalent alternate and mean stress can be written as:

$$
\begin{gathered}
\sigma_{a}^{\mathrm{eq}}=\sqrt{3 J_{2, a}}, \\
\sigma_{m}^{\mathrm{eq}}=J_{1, m} .
\end{gathered}
$$

With the substitution of (4) into (2), Sines criterion can be rephrased as:

$$
\frac{\sigma_{a}^{\mathrm{eq}}}{\sqrt{3} \lambda}+\kappa \frac{\sigma_{m}^{\mathrm{eq}}}{3 \lambda} \leq 1 .
$$

With the definition of load weight factor, the fatigue analysis is implemented with one finite element analysis. With the above constraints, the fatigue life of the structure under the prescribed load can be infinite. Besides, other criteria like Crossland or Dang Van can be readily integrated into the present framework. 


\subsection{Modeling of the joints}

As multi-fastener joint structures, typical models concern panels jointed by single or multiple fasteners. There are two primary aspects in the analysis, i.e., the distribution of joint loads on the fasteners and the stress state around the hole of the panels. However, many factors should be taken into account for the precise simulation, such as the relative displacements of hole centers, fastener deformation, conforming contact between fasteners and member plates as well as effects of fastener clamp-up, friction and out of plane deformation etc.

To tackle the extra complexities and computational difficulties in modeling and simulating of multi-fasteners joints, various techniques are investigated (Chickermane et al. [14], Zhu et al. [15] and Ekh and Schön [9]). For the prediction of the joint load distribution, conventional structural elements are sufficient. But the simplified connection will generate the false stress (usually higher than the true stress state). Real stress state can be obtained when using combined structural elements and 3D element. However, 3D models require nonlinear iteration for contact effect which is difficult to optimize with gradients-based optimization algorithm. To spare the computing effort, combined structural elements are used here.

Figure 2 shows the joint which consists of one fastener and two member sheets. Member sheets are modeled by two layers of shell elements placed in the middle surface of the real sheet. The structure sizes are equivalently derived from the actual size. To accurately reflect the stiffness of the sheets, fastener holes are modeled. The combined model of the fastener consists of two circular surfaces connected with bar elements. Elements representing fasteners and the member sheets are connected by rigid element. Elements of the member sheets around the fasteners within the certain radius are designated as the control volume. It is notable that stresses in the connecting elements are still higher than the real stresses. Thus, fatigue constraints are evaluated in the element in control volume excluding the connecting elements.

\section{Optimization formulation}

The standard topology optimization problem is extended with the fatigue constraints, which can be formulated as:

$$
\begin{aligned}
& \text { find : } \boldsymbol{\rho}=\left(\rho_{1}, \rho_{2}, \ldots, \rho_{i}, \ldots \rho_{n}\right) \text {, } \\
& \min : \quad C_{d}=\frac{1}{2} u^{T} \boldsymbol{K} u, \\
& \text { s.t. : } f=\boldsymbol{K} u \text {, } \\
& V \leq V_{0}, \\
& \frac{\sigma_{a, e}^{\mathrm{eq}}}{\sqrt{3} \lambda}+\kappa \frac{\sigma_{m, e}^{\mathrm{eq}}}{3 \lambda} \leq 1, e=1,2, \ldots m
\end{aligned}
$$

where $\boldsymbol{\eta}$ is the vector of the pseudo-density design variables describing the material distribution in the design domain. The popular Solid Isotropic Material with Penalization interpolation model (SIMP, Bendsøe and Sigmund [16]) is

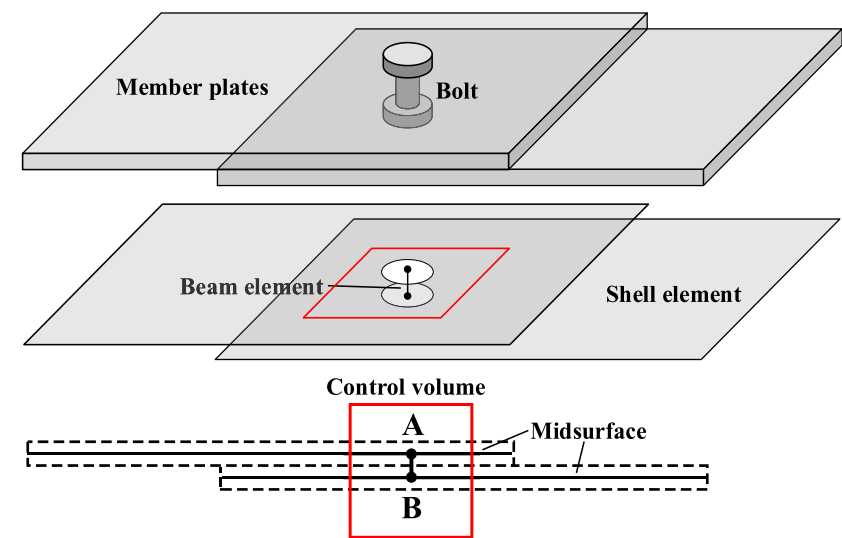

Fig. 2. The model of the multi-fastener joint.

used here. $C_{d}$ is the dynamic compliance. $f$ is the force amplitude vector and $\boldsymbol{u}$ is the corresponding nodal displacement amplitude vector. $\boldsymbol{K}$ is the global stiffness matrix. $V$ is the structural volume and $V_{0}$ is the allowable volume. $m$ is the number of the evaluated elements in the control volume.

\subsection{Issues about fatigue constraints}

The fatigue constraint is implemented in the context of stress-based topology optimization. Thereafter, the numerical problems in stress-based topology optimization are encountered, i.e. stress singularity phenomenon, the local constraint problem and highly nonlinear behavior.

Stress singularity phenomenon is due to the discontinuous nature of the stress constraints, i.e., the stress is discontinuous at zero density and can be very high in the void region. Meanwhile, the discontinuity creates the degenerate design domains. To circumvent the singularity, the $\varepsilon$-relaxation method [17] and $q p$-relaxation method [18] have been proposed. In this paper, the $q p$-relaxation method which chooses a suitable stress interpolate parameter differing from the stiffness penalty factor, is used. In the current context, the stress at the centroid of the iso-parametric element, i.e., the arithmetic mean of the Gauss point stress, is evaluated in the elemental level. According to the SIMP law, the stress in the $e$-th element can be written as: te the fatigue constrain

$$
\begin{gathered}
\sigma_{a, e}^{\mathrm{eq}}=\rho^{p}\left(c_{a} \sqrt{\boldsymbol{u}_{e}^{T} \mathbf{M}_{e} \boldsymbol{u}_{e}}\right)=\rho^{p} \bar{\sigma}_{a, e}^{\mathrm{eq}}, \\
\sigma_{m, e}^{\mathrm{eq}}=\rho^{p}\left(c_{m} \mathbf{H}_{e} u_{e}\right)=\rho^{p} \bar{\sigma}_{a, e}^{\mathrm{eq}}
\end{gathered}
$$

where $\mathbf{M}_{e}$ and $\mathbf{H}_{e}$ is elemental von Mises stress matrix and hydrostatic stress matrix. $\bar{\sigma}_{a, e}^{\mathrm{eq}}$ and $\bar{\sigma}_{m, e}^{\mathrm{eq}}$ is equivalent alternate and mean stress with the initial material properties. With $q p$-relaxation method, the relaxed stress can be expressed as:

$$
\begin{gathered}
\tilde{\sigma}_{a, e}^{\mathrm{eq}}=\rho_{e}^{p-q} \bar{\sigma}_{a, e}^{\mathrm{eq}}, \\
\tilde{\sigma}_{m, e}^{\mathrm{eq}}=\rho_{e}^{p-q} \sigma_{m, e}^{\mathrm{eq}} .
\end{gathered}
$$

where $p>q \geq 1$. 
On the other hand, fatigue is a local phenomenon. Fatigue constraints should be evaluated in every elements in the concerned region which inevitably brings in large amount of constraints. The constraint aggregation algorithm is used as in stress-based topology optimization. The most intuitively method is to merge the local constraints into one global constraint. Besides, P-norm and KS (KreisselmeierSteinhauser function, Duysinx [19], Yang and Chen [20]) are typically used for the aggregation. The drawbacks of globalization are the less control of the local peak value and the high nonlinearity. Further, a clustered approach is proposed. Instead of aggregating all the constraint into a single one, they are gathered into clusters by a specific strategy. When the number of clusters is 1 , the clustered approach degenerates to the global approach and the number cluster equals to that of the original constraints it equivalent to solve the problem directly in a local way. Therefore, the optimized results depend on the cluster strategy.

Follow the basic idea of the clustered approach, only the elements around the fastener holes are defined as control volume and considered. To aggregate the fatigue constraints of the concerned elements, P-norm is introduced here. $\mathrm{P}$-norm is a classic way to approximately achieve the maximum value of a set. Within the control volume, Pnorm of the fatigue constraints can be expressed as:

$$
f=\left(\sum_{e=1}^{n}\left(\frac{\tilde{\sigma}_{a, e}^{e q}}{\sqrt{3} \lambda}+\kappa \frac{\tilde{\sigma}_{m, e}^{e q}}{3 \lambda}\right)^{p n}\right)^{1 / \mathrm{pn}} .
$$

$f$ is the $\mathrm{P}$-norm of the fatigue constraint. The parameter of $\mathrm{p}$-norm $p n$ can be chosen arbitrarily. When $p n$ equals 1 , the p-norm is the sum of the fatigue constraints, and when $p n$ approaches infinity, the p-norm is approximately the maximum value.

$$
\lim _{p n \rightarrow \infty}\left(\sum_{e=1}^{n}\left(\frac{\tilde{\sigma}_{a, e}^{e q}}{\sqrt{3} \lambda}+\kappa \frac{\tilde{\sigma}_{m, e}^{e q}}{3 \lambda}\right)^{p n}\right)^{1 / p n}=\max \{f\} .
$$

Larger value of $p n$ will give an accurate evaluation of the maximum joint load but lead to illness and oscillations (Haftka and Gürdal [21]). In this paper $p n$ is assigned with 6 .

\subsection{Sensitivity analysis}

The sensitivity of the dynamic compliance can be readily derived according to the equilibrium equation and the SIMP law:

$$
\frac{\partial C}{\partial \rho_{e}}=-p \rho_{e}{ }^{p-1} \boldsymbol{u}_{e}^{T} \boldsymbol{k}_{e} \boldsymbol{u}_{e} .
$$

The sensitivity of the fatigue constraint can be expressed as:

$$
\begin{gathered}
\frac{\partial f}{\partial \rho_{e}}=\left(\sum_{e=1}^{n}\left(\frac{\tilde{\sigma}_{a, e}^{e q}}{\sqrt{3} \lambda}+\kappa \frac{\tilde{\sigma}_{m, e}^{e q}}{3 \lambda}\right)^{p n}\right)^{\frac{1}{p n}-1} \\
\sum_{e=1}^{n}\left(\left(\frac{\tilde{\sigma}_{a, e}^{e q}}{\sqrt{3} \lambda}+\kappa \frac{\tilde{\sigma}_{m, e}^{e q}}{3 \lambda}\right)^{p n-1}\left(\frac{1}{\sqrt{3} \lambda} \frac{\partial \tilde{\sigma}_{a, e}^{e q}}{\partial \rho_{e}}+\frac{\kappa}{3 \lambda} \frac{\partial \tilde{\sigma}_{m, e}^{e q}}{\partial \rho_{e}}\right)\right) .
\end{gathered}
$$

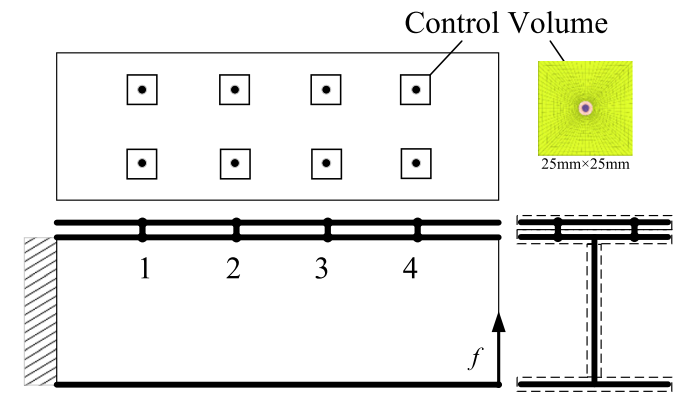

Fig. 3. An I-shape cantilever beam connected with a thin sheet.

Table 1. Mechanical properties of the prescribed material.

Young's module $E$

$210 \mathrm{MPa}$

Possion ratio $\mu$

Fatigue limit for repeated bending $f_{-1}$

$260 \mathrm{MPa}$

Fatigue limit for reversed torsion $t_{-1}$

$130 \mathrm{MPa}$

Ultimate tensile strength $\sigma_{f}$ $580 \mathrm{MPa}$

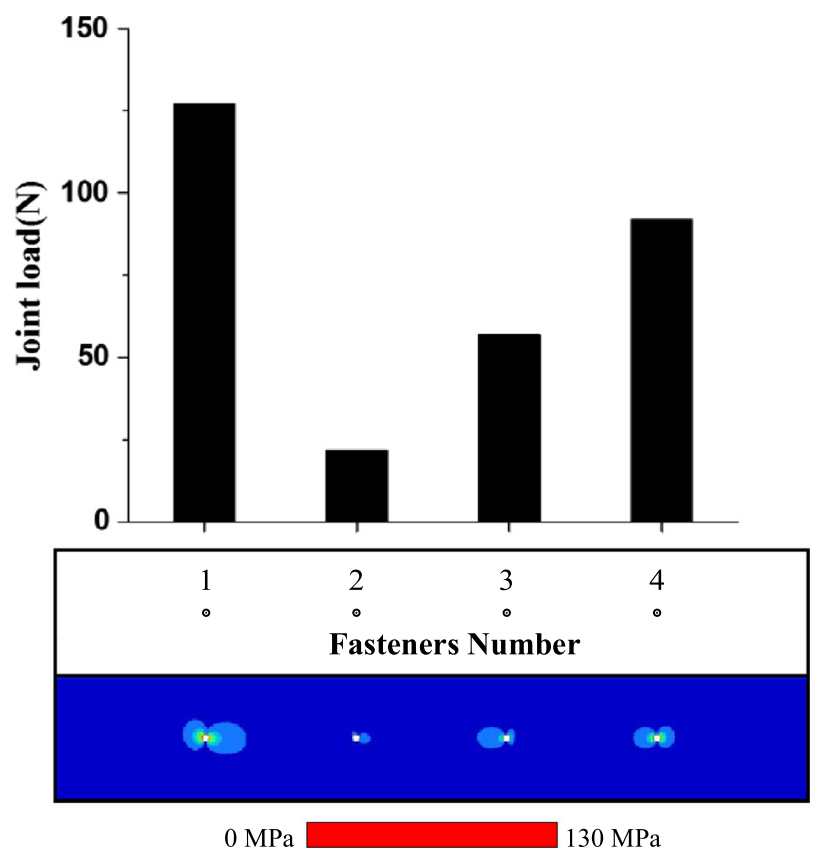

Fig. 4. The stress state and load distribution of the skin.

The sensitivity of the fatigue constraints involve the derivatives of equivalent alternate stress $\bar{\sigma}_{a, e}^{\text {eq }}$ and equivalent mean stress $\bar{\sigma}_{m, e}^{\mathrm{eq}}$, which reads:

$$
\begin{gathered}
\frac{\partial \tilde{\sigma}_{a, e}^{\mathrm{eq}}}{\partial \rho_{e}}=\delta_{\mathrm{ek}}(p-q) \rho_{e}{ }^{p-q-1} \tilde{\sigma}_{a, e}^{\mathrm{eq}}+\frac{\partial \tilde{\sigma}_{a, e}^{\mathrm{eq}}}{\partial \rho_{e}} \rho_{e}{ }^{p-q} \\
\frac{\partial \tilde{\sigma}_{m, e}^{\mathrm{eq}}}{\partial \rho_{e}}=\delta_{\mathrm{ek}}(p-q) \rho_{e}{ }^{p-q-1} \tilde{\sigma}_{m, e}^{\mathrm{eq}}+\frac{\partial \tilde{\sigma}_{m, e}^{\mathrm{eq}}}{\partial \rho_{e}} \rho_{e}{ }^{p-q}
\end{gathered}
$$




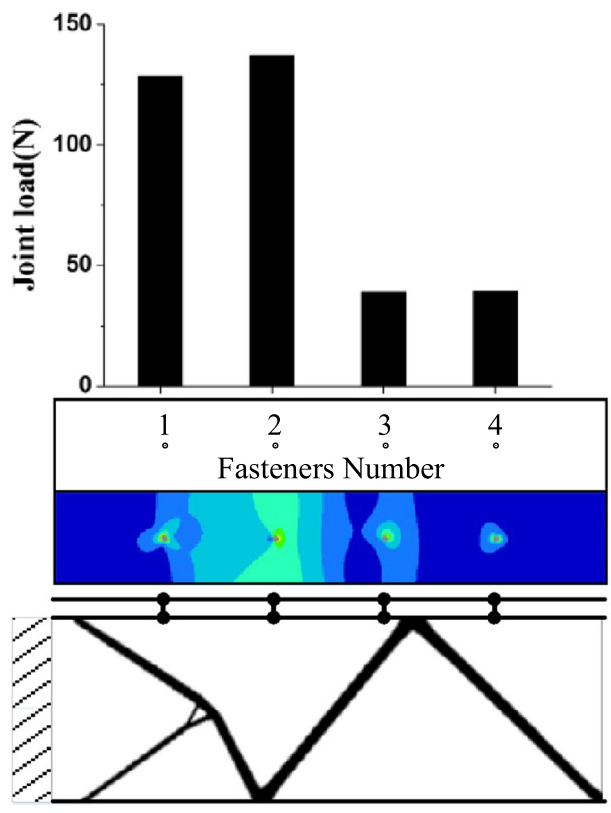

$0 \mathrm{MPa}$

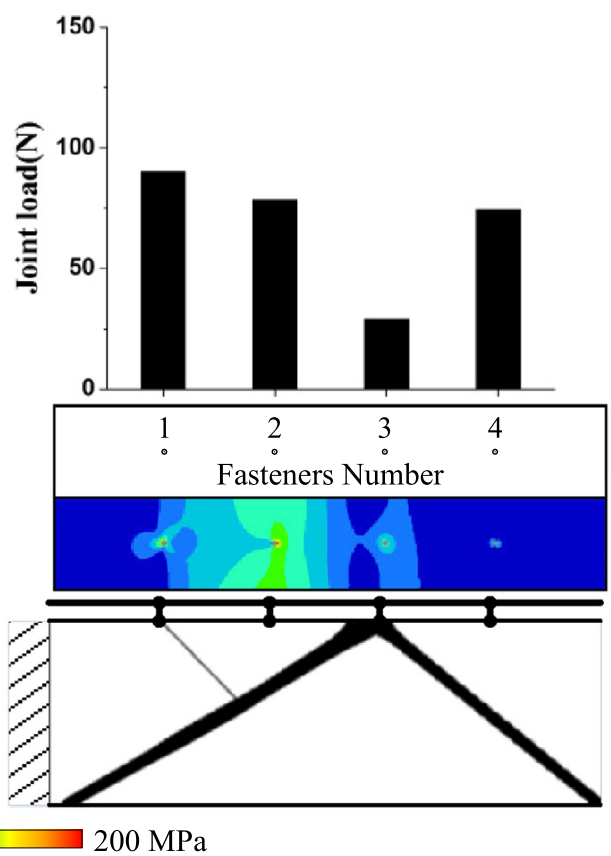

b) topology optimization with fatigue constraint, total dynamic compliance $9.169 \mathrm{KJ}$; maximum displacement 5.11 $\mathrm{mm}$; maximum stress $130 \mathrm{MPa}$; the maximum joint load appears on fastener near the root of the beam and is $90.3 \mathrm{~N}$.

Fig. 5. Optimized configurations of the cantilever beam (a) standard topology optimization, total dynamic compliance 10.21 KJ; maximum displacement $4.60 \mathrm{~mm}$; maximum stress $188.6 \mathrm{MPa}$; the maximum joint load appears on fastener near the root of the beam and is $128.5 \mathrm{~N}$. (b) topology optimization with fatigue constraint, total dynamic compliance $9.169 \mathrm{KJ}$; maximum displacement $5.11 \mathrm{~mm}$; maximum stress $130 \mathrm{MPa}$; the maximum joint load appears on fastener near the root of the beam and is $90.3 \mathrm{~N}$.

where $\delta_{\text {ek }}$ is the Kronecker delta. The derivatives of $\tilde{\sigma}_{a, e}^{\mathrm{eq}}$ and $\tilde{\sigma}_{m, e}^{\mathrm{eq}}$ can be derived by differentiation of $(7)$ :

$$
\frac{\partial \tilde{\sigma}_{m, e}^{\mathrm{eq}}}{\partial \rho_{k}}=-\lambda \frac{\partial \mathrm{K}}{\partial \rho_{k}} U
$$

where $K \lambda=c_{a}\left(\boldsymbol{u}_{e}^{T} \mathbf{M}_{e} \boldsymbol{u}_{e}\right)^{-\frac{1}{2}} \mathbf{M}_{e} \boldsymbol{u}_{e}$

$$
\frac{\partial \tilde{\sigma}_{m, e}^{\mathrm{eq}}}{\partial \rho_{k}}=-\xi \frac{\partial \mathrm{K}}{\partial \rho_{k}} U,
$$

where $K \xi=c_{a} \mathbf{H}_{e}+c_{m} \mathbf{H}_{e}$.

Thus all the sensitivities are derived by the chain rule.

\section{Numerical example}

In the following sections, a numerical example is presented to illustrate the efficiency and validity of the proposed antifatigue topology optimization method. The optimization algorithm of globally convergent method of moving asymptotes (GCMMA [22]) implemented in the generalpurpose design platform Boss-Quattro is used [23]. To prevent the mesh dependency and checkerboard, sensitivities filtering is used with 3 times of the uniform mesh size as the filter radius.

An I-shape cantilever beam connected with a thin sheet by two rows of parallel arranged fasteners is considered. The dimension and the layout of the assembled structure are shown in Figure 3. The thickness of the member panels is uniformity $1 \mathrm{~mm}$, including the skin sheets, the web and the flanges. The gap between the beam flange and sheet is also $1 \mathrm{~mm}$. The web of I-beam is considered as the design domain. The control volume consists of the elements within a $25 \mathrm{~mm} \times 25 \mathrm{~mm}$ square around four fasteners as shown in Figure 3 and are modeled by the proposed method in Section 2.2. The structure is uniformly meshed by $1 \mathrm{~mm} \times 1 \mathrm{~mm}$ quadrilateral shell elements (excluding the elements in the control volume). A point-wise force of $f=4 \mathrm{KN}$ is applied at the lower right corner.

Fatigue analysis is carried out by linear finite element analysis due to the decomposition of the harmonic load and no further treatment is needed. The weight factor $c_{a}$ and $c_{m}$ are set to 0.7 and 0.3 , respectively. To implement the fatigue constraint, the properties of the material are prescribed in the following table.

With the substitution of the mechanical properties in (5), Sines fatigue constraint takes the following form:

$$
0.58 \sigma_{H, a}+0.224 \sigma_{H, m} \leq 130 \mathrm{MPa} .
$$

Based on the result of the finite element analysis under the harmonic load as shown in Figure 1, the maximum vonMises stress of the entire structure is $1887.12 \mathrm{MPa}$ at the connecting points of the fastener models and the maximum von-Mises stress in the loading position is $1038.54 \mathrm{MPa}$. Those dramatically high stresses are theoretically caused by the simplification of the concentrated force and rigid 

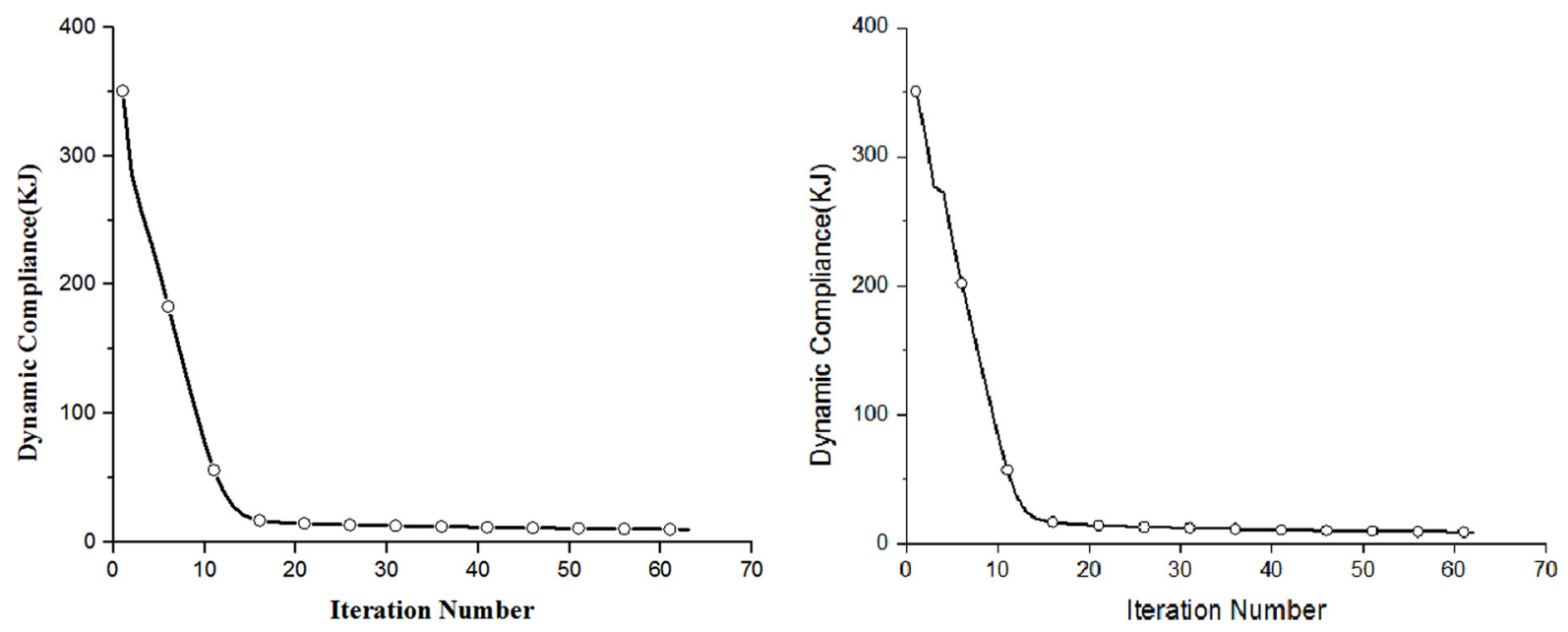

Fig. 6. The iteration histories of the dynamic compliance.

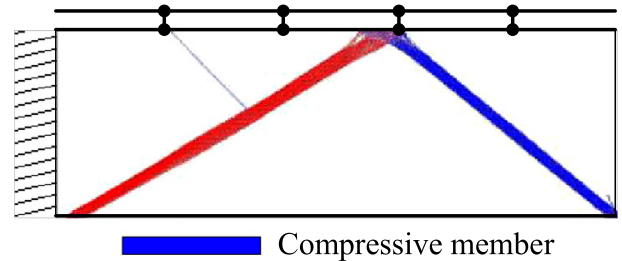

a) standard optimized configuration

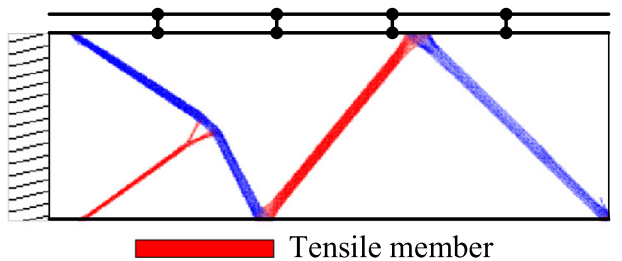

b) optimized configuration with fatigue constraint

Fig. 7. Principal stress of the optimized configuration. (a) standard optimized configuration. (b) optimized configuration with fatigue constraint.

connection. Meanwhile, the maximum von-Mises stress in the upper panel and lower panel are respectively $25.03 \mathrm{MPa}$ and 52.06 $\mathrm{MPa}$, which are relatively close to the true stress states. Hence, only the stress states of the control volume are considered. In Figure 4, the Sines equivalent stress on the skin sheet along with the load distribution on the fasteners are shown. In the initial configuration, the maximum Sines equivalent stress is 129.3 MPa and satisfies the fatigue constraint. The maximum fasteners joint load is $90.3 \mathrm{~N}$.

Here, a standard topology optimization is carried out to minimize the dynamic compliance. For a light-weight design, the allowable structural volume is assigned as $30 \%$ of the design domain. Subsequently, the fatigue constraint is imposed and evaluated in the elements within the control volume. Two optimized designs are shown in Figure 5 and significant differences can be observed. In the configuration of (a), the maximum Sines stress is $188.6 \mathrm{MPa}$ which is higher than the allowable fatigue constraints. On the contrary, the P-norm of the stress state of the latter configuration satisfies the fatigue constrains, and the maximum stress is $130 \mathrm{MPa}$. The stress states and the load distributions are plotted in Figure 5. It can be seen that in the configuration with fatigue constraint. Figure 6 shows the convergence curve of the dynamic compliance. After 62 and 61 iteration, both the optimization converged very well.
To give the insight of how fatigue constraints affect the optimized configuration, the principal stress maps of two corresponding results are plotted in Figure 7 . The result in Figure 7a apparently has better stiffness with more supporting branches near the fixed end. However, better stiffness generates more severe stress concentration in the structure especially around the joint areas. In the configuration with the fatigue constraint, two main branches transfer the load directly and a minor branch appears to decrease stresses on fastener near the root of the beam. The loss of stiffness is considered as trade-off for the strength.

\section{Conclusion}

In this paper, failure of the fastener jointed structure is considered and solved in the context of stress based topology optimization. The joints are modeled as short beam elements. The idea of control volume is introduced to evaluate the stress state around the fasteners to eliminate the unreal stress concentration. The fatigue analysis is based on the static finite element analysis because of the decomposition of the harmonic load. The Sines fatigue criterion is used to transfer the fatigue constraint into stress constraint which can be steadily handled. A numerical example is tested and optimization results are 
compared with standard topology optimization design. It is shown that the fatigue behavior is strongly affected by the structural layout and the load carrying path. With optimized distribution of structural stiffness, Sines equivalent stresses in the control volume are perfectly controlled by the prescribed upper bound. The proposed method provides a significant guidance to designers and engineers in the engineering applications.

\section{References}

1. B. Desmorat, R. Desmorat, Comptes Rendus Mec. 336, 448 (2008)

2. M. Mrzyglod, Eur. J. Physiol. 302, 803 (2010)

3. M. Mrzyglod, P. Zielinski, Struct. Multidiscip. Optim. 33, $161(2007)$

4. N. Kaya, I. Karen, F. Öztürk, Mater. Des. 31, 3008 (2010)

5. K. Sherif, W. Witteveen, K. Puchner, H. Irschik, AIAA J. 48, 1339 (2010)

6. E. Holmberg, B. Torstenfelt, A. Klarbring, Struct. Multidiscip. Optim. 50, 207 (2014)

7. M. Collet, M. Bruggi, P. Duysinx, Struct. Multidiscip. Optim. 55, 1 (2016)

8. Y. Zhang, D. Taylor, Finite Elem. Anal. Des. 37, 1013 (2001)

9. J. Ekh, J. Schön, Compos. Struct. 82, 245 (2008)

10. G. Bianchi, G.S. Aglietti, G. Richardson, Technology (2007)
11. A. Oinonen, P. Tanskanen, T. Björk, G. Marquis, Struct. Multidiscip. Optim. 40, 597 (2010)

12. C. Poon, Y. Xiong, Design of bolted joints for composite structures (Woodhead Publishing Limited, UK, 1995), pp. 629-636.

13. R.L. Norton, Machine design: an integrated approach (2000)

14. H. Chickermance, H.C. Gea, R.J. Yang, C.H. Chuang, Struct. Optim. 17, 140 (1999)

15. J.-H. Zhu, J. Hou, W.-H. Zhang, Y. Li, Struct. Multidiscip. Optim. 50, 561 (2014)

16. M.P. Bendsøe, O. Sigmund, Topology optimization: theory, methods and applications (2003)

17. G. Cheng, X. Guo, Struct. Optim. 13, 258 (1997)

18. M. Bruggi, Struct. Multidiscip. Optim. 36, 125 (2008)

19. P. Duysinx, O. Sigmund, New developments in handling stress constraints in optimal material distributions, in: Proceedings of 7th AIAA/USAF/NASA/ISSMO Symposium on Multidisciplinary Design Optimization, Saint Louis, Missouri. USA: American Institute of Aeronautics and Astronautics, 1998.

20. R.J. Yang, C.J. Chen, Struct. Multidiscip. Optim. 12, 98 (1996)

21. R.T. Haftka, Z. Gürdal, Elements of structural optimization (Kluwer Academic, Dordrecht, Netherlands, 1992)

22. K. Svanberg, The method of moving asymptotes - a new method for structural optimization, in International Journal for Numerical Methods in Engineering (New York, 1987), pp. 359-373.

23. Y. Radovcic, A. Remouchamps, Struct. Multidiscip. Optim. 23, $140(2002)$

Cite this article as: Jie Hou, Jihong Zhu, Jie Wang, Weihong Zhang, Topology optimization of the multi-fasteners jointed structure considering fatigue constraints, Int. J. Simul. Multidisci. Des. Optim. 9, A4 (2018) 